\title{
Integración Social y Economía de Comunión
}

\author{
Howard Richards*
}

\author{
Nivel: Comunicado \\ Recibido: 28 de mayo de 2012 \\ Aprobado: 11 de julio de 2012
}

\begin{abstract}
Resumen
Sugiero asumir la economía de comunión como una forma de integración social. Propongo entender la integración social enfocando la inclusión en un orden social normado. Entiendo la "norma", concepto clave de ambas, en un sentido humanista. Planteo que la ciencia incorpore dicho sentido humanista en su teorización y en sus metodologías de investigación, porque así la ciencia se hace capaz de contribuir a la reconstrucción de la economía.

En cuanto al método de esta ponencia, este se basó en una serie de reflexiones sobre palabras y frases claves, los que fueron puestos en su trayectoria histórica, anunciando sus varios sentidos y usos en las ciencias, para lo cual se recurre a datos históricos y hallazgos de estudios empíricos.
\end{abstract}

Palabras clave: Integración social, Economía de Comunión, racionalidad económica, orden social

\begin{abstract}
I suggest taking the economy of communion as a form of social integration. I suggest understanding social integration focusing on a regulated social order. I understand the "norm", a key concept of both in a humanistic sense. I argue that science incorporates such humanistic view in their theorizing and research methodologies, because science may contribute to the reconstruction of the economy. As for the method of this paper, this was based on a series of reflections on key concepts and phrases, which were placed in its historical period, announcing its various meanings and uses in sciences, making use of historical data and empirical findings.
\end{abstract}

Keywords: Social Integration, Economy of Communion, Economic Rationality, Social Order

* Licenciado en filosofía, University of Yale, Doctor en Leyes, University of Stanford, Doctor en Filosofía, University of California Santa Bárbara, Doctor en Educación, University of Toronto. Docente de la Universidad de Santiago de Chile y Universidad de Buenos Aires. howardri00@yahoo.com 


\section{Introducción}

\section{La integración social}

Inicio esta exposición con la "integración social". Su uso actual en las ciencias sociales deriva de la obra pionera de Emile Durkheim (1858-1917), comenzando con su tesis doctoral en la Universidad de París de 1893. En su obra la frase "integración social" es sinónima con "solidarité", y especialmente con lo que Durkheim llama "solidarité organique", que es el tipo de solidaridad propia de una sociedad moderna.

Hoy en día es frecuente usar la frase "integración social" para significar en un primer momento la presencia de distintos tipos de personas en los mismos lugares. Se dice, por ejemplo, que las escuelas públicas deben promover la integración social para educar tanto a niños pobres como a niños ricos en las mismas aulas. Sin duda el trasfondo de proponer la integración de distintos tipos de alumnos en el mismo espacio, apuesta a que así se conocerán mejor y de su conocimiento mutuo va a surgir una cultura más unificada.

Seguramente "integración social" no sólo se refiere a la presencia de los distintos estamentos sociales en las mismas escuelas, sino que también a los resultados esperados de su co-presencia. Se dice también que el actual sistema educativo chileno promueve la segmentación social, en vez de la integración social, debido a que separa físicamente a los estudiantes de las distintas clases sociales en varias escuelas. Se supone que el hecho de educarse en distintos lugares con distintas cualidades académicas, es un factor en la continuación de las divisiones sociales que caracterizan el país.

No niego la validez del discurso común que acabo de mencionar. Sin embargo, quiero destacar que este discurso no capta ni la totalidad ni el significado principal de la "integración social". Es en este sentido que yo quiero rescatar y valorar los aspectos de la propuesta de Durkheim. 
Como llevo dicho, Durkheim escribía de "solidarité sociale" como sinónimo de "integración social". Se puede extraer de Durkheim, principalmente de su estudio del suicidio y de su estudio sobre la división social del trabajo, la siguiente definición positiva de "integración social"1.

A. Las pasiones de los individuos son reguladas por símbolos culturales compartidos.

B. Los individuos son unidos a las instituciones por rituales y rutinas de la vida diaria en las cuales todos participan, y que dan fuerza a su normatividad.

C. Las acciones son reguladas y coordinadas por normas sociales y por estructuras políticas legítimas.

D. Las desigualdades existentes son consideradas legítimas. Corresponden a distintos aportes a la sociedad cuyos méritos son generalmente reconocidos ${ }^{2}$.

Anthony Giddens ha observado que los principales conceptos de la sociología, sean de la tendencia durkheimiana, sean de la tendencia marxista, sean de la tendencia weberiana, sean de otra tendencia, derivan de las distintas formas en las cuales los grandes pioneros de las ciencias sociales han dado cuenta del auge de la modernidad ${ }^{3}$. El concepto de "integración social" de Durkheim calza con esta observación de Giddens.

La modernización consiste en gran parte en una creciente división del trabajo, una creciente especialización de funciones sociales, una creciente dependencia de las personas para conseguir su pan diario de los mercados y de las relaciones entre las personas reguladas por contratos. Las sociedades pre-modernas, iden-

1 (Durkheim 1897, 1893)

2 Jonathan H. Turner, "Durkheim's Theory of Integration in Differentiated Social Systems". The Pacific Sociological Review. Tomo 24, No. 4 (octubre 1981) pp. 379391.

3 Anthony Giddens, Capitalism and Modern Social Theory: An Analysis of the Writings of Marx, Durkheim, and Max Weber: Cambridge UK: Cambridge University Press, 1971. (Ver la Introducción) 
tificadas por Durkheim como arcaicas, tienden a ser, en cambio, societés segmentées, donde hay más artesanos relativamente autosuficientes que trabajadores altamente especializados, y donde la familia extendida, el clan, las obligaciones recíprocas reguladas por normas gobiernan muchos aspectos de la cooperación necesaria para vivir. Ahora, en la modernidad, las necesidades de la vida se consiguen típicamente por los mercados y los contratos mercantiles. En este sentido, la pobreza urbana es más moderna y más dura que la pobreza campesina, lo que calza con el hecho observado en las escuelas chilenas, que el desacato de normas elementales por parte de los alumnos, es más severo en la ciudad que en el campo.

Durkheim sacó la misma conclusión que ha sacado recién el sociólogo chileno Eugenio Tironi en su libro El Sueño Chileno ${ }^{4}$. Es la misma conclusión que sacó de sus estudios históricos Karl Polanyi ${ }^{5}$. La modernidad padece de una fuerte tendencia a la desintegración social ${ }^{6}$. Sus principios mercantiles rectores tienden a excluir a quienes no tienen nada que vender que sea apetecido en el mercado, y tienden a atomizar las relaciones sociales incluso en los casos de las personas exitosas en el mercado. Por lo tanto, esto es el tema de los últimos capítulos de De la division du travail social: la integración de una sociedad moderna comercial depende del cultivo de complementos necesarios del mercado. Depende del cultivo de una serie de instituciones que obedecen a otras lógicas. Depende de relaciones humanas solidarias que no sean mercantiles, o que sean una síntesis mercantil y solidaria a la vez.

4 Eugenio Tironi, El Sueño Chileno. Comunidad, familia, y nación en el bicentenario. Santiago: Editorial Taurus, 2005.

5 Karl Polanyi, La Gran Transformación. Los orígenes políticos y económicos de nuestro tiempo, Fondo de Cultura Económica (1944), Fondo de Cultura Económica, México, 2003.

6 Juan Cassasus, "Los sistemas de ideas, el poder, y la identidad", en Sergio Martinic y Marcela Prado (eds.). Economía Política de las Reformas Educativas en América Latina. Santiago: CIDE/OREAL, 2001 pp. 341-342). 
El más famoso de los libros de Durkheim es su estudio empírico de las tasas de suicidio en la Europa de su época 7 . Ahí él pudo mostrar que a altos niveles de integración social corresponden bajos niveles de suicidio, y que a altos niveles de desintegración social corresponden altos niveles de suicidio. Entonces, los suicidios son mayores entre los más pobres y los más ricos, por estar ambos, por razones distintas, fuera de la densa trama de normas que suelen regular las vidas de las capas medias.

Los suicidios son menores entre los sin fe, vale decir entre quienes no encuentran sentido en su vida. La religión (según los datos de Durkheim, especialmente las religiones católicas y judías) disminuye la tasa de suicidio. Las familias unidas y extendidas corresponden a menor tendencia a la auto-destrucción. Las personas solas frente a la vida tienen más tendencia a matarse.

Durkheim destaca que el suicidio es un fenómeno de la modernidad, que rara vez se encuentra en las comunidades arcaicas, menos en algunos casos excepcionales como los son las prácticas de tribus que usan el suicidio ritual como una forma de sacrificio ceremonial ante sus dioses. En La division du travail social, Durkheim hasta dibujó mapas de la Europa de su época, mostrando que en las regiones más modernizadas la tasa de suicidio era mayor, mientras en las regiones más tradicionales la tasa de suicidio era menor ${ }^{8}$.

$\mathrm{Al}$ recomendar el uso del concepto durkheimiano de integración social como parte de un paradigma ético, y al recomendar el logro de mayores niveles de ella como meta de las políticas públicas, de los movimientos e instituciones civiles, no quiero declararme incondicional de Durkheim. No me sumo a las filas de toda antropología o sociología de inspiración durkheimiana, ni

7 Emile Durkheim, Le suicide. París: Libraire Felix Alcan, 1930 (primera edición 1897).

8 Emile Durkheim, De la division du travail social. París: Felix Alcan, 1902 (primera edición 1893). 
menos a los abordajes que se han designado estructural-funcionalistas ${ }^{9}$-sin adherir a esta escuela, sigo pensando que a menudo la palabra "estructura" y la palabra "función" sean útiles, este último como elemento de un paradigma ético-. No niego que haya hallazgos válidos encontrados por investigadores quienes se han dedicado adrede a mostrar que un "modelo consensual durkheimiano" describe el mundo real menos que "un modelo conflictivo marxista"10, ni niego que haya hallazgos válidos encontrados por quienes se han dedicado a mostrar que el pensamiento durkheimiano peca por menospreciar la creatividad de los agentes humanos $^{11}$. Es más. La agencia humana es una viga maestra de un paradigma ético.

Sin embargo cabe mencionar que existe un acervo considerable de estudios empíricos que muestran que efectivamente los problemas tienden a resolverse donde hay más integración, y a agudizarse donde haya menos ${ }^{12}$. Las ideas de integración y desintegración han servido para aclarar otros fenómenos más allá del suicidio y de la marginalidad económica, como los son el divorcio ${ }^{13}$, la delincuencia ${ }^{14}$ y el abuso de sustancias como el alcohol ${ }^{15}$. Se ha podido mostrar que las enfermedades mentales

9 Por ejemplo, A.R. Radcliffe-Brown, Structure and Function in Primitive Societies. New York: Free Press, 1965.

10 Por ejemplo, Claude Meillassoux, Maidens, Meal, and Money. Cambridge: Cambridge University Press, 1981.

11 Verónica Edwards, Los Sujetos y la Construcción Social del Conocimiento Escolar en Primaria: un estudio etnográfico. Segunda edición. Santiago: Ediciones PIIE, 1990. Edwards ha mostrado cómo una escuela es una construcción social construida principalmente por los alumnos. Jean Piaget, explícitamente criticando a Durkheim, mostró cómo los niños inventan sus propios juegos en su Le jugement morale chez l'enfant. París: Presses Universitaires de France, 1932.

12 p.ej. K.D. Breault, "Suicide in America, an Empirical Test of Durkheim's Theory of Religious and Family Integration", American Journal of Sociology. Tomo 93, No. 2 (Nov. 1986) pp. 628-656.

13 Alan Booth, John N. Edwards, David R. Johnson, "Social Integration and Divorce", Social Forces Tomo 70, No. 1 (Sep. 1991), pp. 207-224.

14 Clayton A. Hartjen, "Delinquency, Development, and Social Integration in India", Social Problems. Tomo 29, No. 5 (Jun. 1982), pp. 464-473.

15 Barry Glassner y Bruce Berg, "How Jews Avoid Alcohol Problems", American Sociological Review. Tomo 45, No. 4 (agosto 1980), pp. 647-664. 
ocurren con mayor frecuencia entre las personas en situaciones precarias y solas. La enfermedad mental es menos frecuente entre quienes gozan de la seguridad económica, de una familia y de una comunidad unida ${ }^{16}$.

En este contexto ha resultado útil un análisis de cuatro tipos de integración social propuesto por Werner Landecker en 1951, a saber: la integración funcional -o sea, el papel (o en el caso de desintegración, la falta de papel) en la vida económica y material de la sociedad-; la integración cultural, es decir, ser parte de un grupo social que comparte normas, símbolos, sentido de vida; la integración normativa, o sea, la existencia de sanciones e incentivos que hacen eficaces las normas culturales como reguladores de la vida, y la integración comunicacional, entendiéndola como un fluido intercambio de significados entre las personas que comparten una integración cultural ${ }^{17}$.

Evidentemente, la integración concebida como la corrección de los excesos de un modernismo desenfrenado, corre el riesgo de fomentar los vicios típicos de las sociedades arcaicas, como son el patriarcado, la xenofobia y el control sofocante del individuo. Entonces, sugiero que estamos llamados a conjurar este riesgo. En este sentido conviene considerar el pensamiento comunitario de Amitai Etzioni, quien destaca que en nuestras complejas sociedades actuales, el individuo tiene opciones entre varias comunidades y puede pertenecer a varias a la vez. De este modo obtiene las ventajas de la pertenencia, sin tropezar con las limitaciones que caracterizan a las culturas arcaicas y tradicionales ${ }^{18}$.

16 G. Nelson, J. Lord, y J. Ochocka, Shifting the Paradigm in Community Mental Health: towards empowerment and community. Toronto: University of Toronto Press, 2001.

17 Werner S. Landecker, "Types of Integration and their Measurement", American Journal of Sociology, Tomo 56, No. 4 (enero 1951), pp. 332-340.

18 Amitai Etzioni, The Spirit of Community: The Reinvention of American Society. New York: Simon and Schuster, 1994. Véase también otras obras del mismo autor. 


\section{Economía de Comunión}

La economía de comunión es una forma de integración social. Por un lado, es la inclusión, y, por otro lado, es la subordinación de la vida económica a una normatividad solidaria.

A partir de ahí podemos reseñar que la economía de comunión, en un sentido estricto de la palabra, es una serie de formas de organización económica promovidas por el Movimiento Eclesial de los Focolares. Fue lanzada por Chiara Lubich, fundadora del movimiento, en Brasil en $1991^{19}$. Tiene como objetivo contribuir a sanar la creciente diferencia entre ricos y pobres. Propone practicar un amor que «no se refiere sólo a los individuos, ni es limosna o voluntariado, sino que se refiere a la cultura, las estructuras y los dinamismos de la sociedad. El amor llega a ser el criterio para la transformación del mundo ${ }^{20}$. Uno de sus lemas fundamentales es "que todos sean uno".

En 1944, Chiara Lubich y unas pocas seguidoras en la ciudad de Trento, Italia, ciudad recién devastada por un bombardeo aéreo, arrendaron un pequeño departamento desde el cual se dedicaban al alivio espiritual y material de los damnificados. Es el primer focolar. En 1948, en la misma ciudad, se abre el primer focolar masculino ${ }^{21}$. En el mismo año se inicia como parte del movimiento focolar, el Movimiento Familias Nuevas que integra casados, habiendo sido los primeros focolares exclusivamente solteras y solteros.

Estas experiencias suscitan el interés de algunos jóvenes marxistas. El diálogo con ellos y otras personas sin un credo, se desarrolla seguidamente. Hoy en día el movimiento "economía de

19 Chiara Lubich, La Economía de Comunión, Historia y Profecía. Buenos Aires: Editorial Ciudad Nueva, 2003.

20 Mensaje de cardenal Enni Antonelli, arzobispo de Florencia, 6 noviembre 2006, disponible en español en www.catholic.net/empresarioscatolicos.

21 www.focolares.org.ar 
comunión" organiza empresas que dedican sus utilidades a fines sociales $^{22}$. Es una red de pequeñas y medianas empresas abocadas a los principios éticos de las religiones del mundo, pero no son solamente cristianos ${ }^{23}$. Aunque comenzó como movimiento católico, desde los años 1950, ha contado también con adherentes de otras tradiciones. En la actualidad está presente en 180 países.

La pobreza evangélica, presente no solamente en tradiciones católicas como la franciscana, sino también en otras religiones, llega a ser menos un fin en sí, y más un medio para reducir la desigualdad e incluir a los excluidos. "El corazón del problema", según la fundadora Chiara Lubich, es el deseo de tener bienes propios en vez de sentirse conectado con otros como en una familia $^{24}$.

Aquí encontramos el mismo asunto que trató Durkheim desde un punto de vista menos espiritual. El principio organizador de las antiguas sociedades humanas era, nos dice Durkheim, el parentesco, el sentido de familia extendida. Si en la modernidad nos acosa el problema de la anomia, con su secuela de problemas como el suicidio, es porque la modernidad se aparta de este principio organizador. El movimiento de los Focolares y sus hermanos de las distintas religiones del mundo, recuperan el principio de la convivencia humana e intentan aplicarlo al mundo actual; y se inspiran en otra institución casi tan antigua como la familia, y que es la religión. El objetivo es sencillo: llegar a la meta de que no haya más necesitados, que todos tengan suficiente para vivir ${ }^{25}$.

Las empresas que forman parte del movimiento operan en el mercado libre y se conforman con las leyes vigentes. Dividen sus

22 Lorna Gold, "The Economy of Communion: a case study of business and civil society partnership for change". Development in Practice. Vol 14 (2004), pp. 633-44. 23 Lorna Gold, "The Roots of the Focolare Movement's Economic Ethics", Journal of Markets and Morality. Vol. 6 (2003) pp. 143-59.

24 Id. p. 147.

25 Chiara Lubich, "Erano un cuor solo e una anima sola", Amico Serafico (1948), pp. 236-37. 
ganancias en tres partes: una parte va a los pobres, una parte se reinvierte en la empresa y una parte se dedica a la evangelización en el sentido de formación espiritual. La fe es una parte integral de la cultura corporativa, inspira la honestidad, la contabilidad correcta y no "cocinada", las prácticas verdes, el tratamiento de los compañeros en el trabajo como hermanos y hermanas cocreadores, y así sucesivamente.

A fin de situar un movimiento actual como lo es la Economía de Comunión en la historia de las relaciones entre economía y religión, nos referimos a los estudios históricos de Max Weber. Para Weber es típico en las sociedades pre-modernas, y en todas las sociedades aun cuando sean en principio seculares, que la religión se plasme en una ética económica.

De la ética económica de una religión, se siguen, nos enseña Weber, normas acerca del trabajo, normas acerca de la riqueza y de las posesiones, normas sobre el comercio y la industria, normas acerca de las finanzas, normas sobre relaciones con otros actores económicos, normas sobre las innovaciones y cambios, y normas acerca de las relaciones con quienes carecen de recursos económicos ${ }^{26}$.

\section{Estatus científico de la norma}

La norma ética resulta ser una categoría necesaria para comprender no solamente la economía de comunión en sentido estricto, sino también cualquier movimiento que enfoca la reconstrucción de la economía como un proyecto ético de integración social, como los son la economía solidaria ${ }^{27}$, la economía social ${ }^{28}$, la so-

26 Richard Swedberg, Max Weber and the Idea of Economic Sociology. Princeton: Princeton University Press, 1998. p. 138.

27 Ver Luis Razeto, Las Empresas Alternativas. Montevideo: Nordam, 2000.

28 Ver Jose Luis Coraggio, De la Emergencia a la Estrategia. Buenos Aires: Espacio Editores, 2004. 
cioeconomía ${ }^{29}$, ciertas formas de socialismo democrático ${ }^{30}$ y algunas formas de capitalismo responsable ${ }^{31}$.

Entonces surge la pregunta: ¿Cómo la norma calza con la investigación científica? Sugiero que surge también un tema perenne de la filosofía de la ciencia y, especialmente, de la filosofía de la biología, que es el tema de la teleología, de las finalidades. Por ejemplo, en el caso de la economía de la comunión se plantea una finalidad, a saber: llegar a la meta de que no haya más necesitados, que todos tengan suficiente para vivir. Se plantea también una norma, a saber: dividir las utilidades en tres partes, una para los pobres, una para re-invertir, una para la formación espiritual. La reflexión sobre la experiencia y la investigación científica, son relevantes para evaluar en qué medida la norma funciona para cumplir la finalidad.

Eso no quiere decir que las normas deban ser siempre cambiantes, de acuerdo a los vaivenes de sus cambiantes evaluaciones de funcionamiento. Al contrario, es conveniente que las normas sean estables y que su paulatina revisión sea pausada para mejorar su funcionamiento ${ }^{32}$. Por otra parte, sí vale decir que para realizar investigaciones sociales se debe contar con una categoría de "función" o "finalidad".

Humberto Maturana y Francisco Varela han examinado la vieja problemática de la "función", "causas finales" o "teleología", a la luz de los cambios paradigmáticos en las ciencias biológicas $^{33}$. Destacan que las verdades de la cibernética no dependen

29 Ver J. Pérez Adán, Socioeconomía. Madrid: Editorial Trotta, 1997. Ver también las obras de Amartya Sen y las de Amitai Etzioni.

30 Ver Howard Richards, Solidaridad, Participación, Transparencia: conversaciones sobre el socialismo en Rosario, Argentina. Rosario: Fundación Estévez Boero, 2008.

31 Ver C.K. Prahalad, La Fortuna en la Base de la Pirámide. Madrid: Granica, 2005.

32 Howard Richards, "Deference", International Journal of Ethics. Volume 74 (1964), pp. 135-142.

33 Francisco Varela and Humberto Maturana, "Mechanism and Biological Explanation", Philosophy of Science. Vol. 39 (1972) pp. 378-382. 
de sus aplicaciones físicas. Citan a Norbert Wiener: Prima "la información, no la materia y no la energía. Ningún materialismo que no reconozca esto pueda sobrevivir en la actualidad" ${ }^{\prime 34}$. Sin embargo, en cuanto a la biología, afirman Varela y Maturana: "los sistemas vivientes son máquinas de una o varias clases definidas". Vale decir, el cambio paradigmático que destaca la información por sobre la materialidad, en las ciencias biológicas como en otras ciencias, deja ilesa una filosofía mecanicista de la biología. El cambio es que el mecanismo mismo se define en términos de relaciones informáticas, y no por su forma material.

En forma consecuente, Maturana y Varela mantienen la tradición de rechazar el vitalismo y la teleología en las ciencias biológicas. Comparan dos enunciados:

T1 La función del ADN es codificar las proteínas.

T2 ADN codifica las proteínas.

Sacan la conclusión, después de revisar varios argumentos pro y contra el reconocimiento de la categoría "función" en biología, que T2 es suficiente. Dice todo lo que hay que decir. Se puede hacer biología sin hablar de funciones.

Varela y Maturana citan los argumentos analizados por el filósofo Morton Beckner en pro y contra de la teleología, y en pro y contra del uso de un concepto de "función" en las ciencias ${ }^{35}$. Las conclusiones de Beckner no son tajantes ni a favor de la categoría de "función" ni contra ella. Más importante, desde el punto de vista de la promoción de la integración social y la economía de comunión, Beckner interpreta las motivaciones de quienes han luchado contra cualquier forma de teleología en las ciencias. Escribe Beckner: Quienes rechazan la categoría de función "quieren

34 Id. p. 379.

35 M. Beckner, The Biological Way of Thought. New York: Columbia University Press, 1969; M. Beckner, "Function and Teleology", Journal of the History of Biology. Vol. 2 ( 1969), pp. 151-164. 
defenderse contra varias cosas: la importación en la ciencia de contenidos teológicos o metafísicos que no sean verificables, la idea de que las metas de alguna manera actúan a favor de su propia realización, y la idea de que los sistemas biológicos requieren para su explicación conceptos que no se asemejen a nada utilizado en las ciencias físicas" ${ }^{\prime 36}$.

Sugiero lo siguiente. El interés de aquella ciencia social que se dedica a apoyar los procesos de integración social no es precisamente la explicación. La explicación a menudo es útil, pero en fin de cuentas lo que nos interesa es que las instituciones funcionen mejor $^{37}$. Sea lo que sea, la conveniencia de una categoría de "función" para otras ciencias, es indispensable para el apoyo científico al desarrollo local. Nos importan dos de las motivaciones que cita Beckner para resistir la teleología. Efectivamente debemos tener conceptos distintos de los de las ciencias físicas. Aunque la naturaleza no tenga finalidades, los seres humanos y nuestras instituciones sí las tienen. Aunque la religión y la metafísica no sirvan en las explicaciones de las ciencias naturales, las creencias de tipo religioso o metafísico que tenga la gente, sirven de mucho en las explicaciones de los sociólogos. En un caso dado pueden ser funcionales o anti-funcionales.

Con mayor razón sirve para la integración social dar un sentido humano a las normas. Según Durkheim, una norma es una regularidad observada en la conducta humana que cuenta con la sanción de la autoridad ${ }^{38}$. Cabe matizar su concepto a la luz de los hallazgos que encuentran que a menudo los grupos humanos mantienen una regularidad de conducta en el plano ideal, aun-

\footnotetext{
36 "Function and Teleology" p. 164.

37 En su famoso libro Erkenntnis und Interesse, Jurgen Habermas analiza tres intereses de la ciencia: comprender (Verstehen), explicar (Erklären) y emancipar. Sugiero un cuarto: mejorar las instituciones. (Jurgen Habermas, Conocimiento e Interés. Buenos Aires: Amorrortu, 1975).

38 Emile Durkheim, De la division du travail social. París: Felix Alcan, 1902, (primera edición 1893).
} 
que sea infrecuente su cabal cumplimiento en la práctica. Cabe también ampliarlo siguiendo el análisis de Hart del concepto de "regla"39. Así una "regla" o "norma" es: (1) una regularidad observada en la conducta humana, aunque sea en algunos casos solamente una regularidad ideal poco cumplida, (2) una licencia para evaluar y criticar, y llegado el caso castigar, a quienes la violan, (3) una guía que los seres humanos utilizan para monitorear y auto-regular su propia conducta. En este tercer punto de Hart, entran los elementos clásicos de la ética de Aristóteles: la deliberación (prohairesis), la decisión, la acción ${ }^{40}$.

Para trabajar con las normas, y por ende para aportar a la construcción de mayores niveles de integración social, la ciencia debe contar con una serie de elementos humanos que no sean propios de las ciencias naturales como, por ejemplo: finalidades, evaluación, deliberación, decisión y otros. En fin, debe contar con lo que los filósofos británicos llaman una teoría de acción humana ${ }^{41}$.

Al complicar la ciencia con otro tipo de causalidad, la causalidad humana en la cual las deliberaciones y decisiones de las personas son causas, y en la cual las normas que constituyen las instituciones son causas, creo que cumplimos con el espíritu del pensée complexe de Edgar Morin. Morin destaca que la ciencia ya no trabaja solamente con la antigua causalidad lineal ${ }^{42}$. Ahora también se reconoce la causalidad recursiva y la causalidad cir-

39 H.L.A. Hart, The Concept of Law. Oxford: Clarendon Press, 1962.

40 G.E.M. Anscombe y Tomas Abraham han planteado que difícilmente podamos hablar de ética sin invocar categorías aristotélicas. G.E.M. Anscombe, "Modern Moral Philosophy", Philosophy. Vol. 33 (1958), pp. 1-19; Tomás Abraham, Batallas Éticas. Buenos Aires: Nueva Visión, 1995.

41 Margaret Archer, Being Human: the problem of agency. Cambridge: Cambridge Univesity Press, 2000; Stuart Hampshire, Thought and Action. New York: Viking Press, 1960.

42 Edgar Morin, Introductión a la pensée complexe. París: ESF, 1990. Morin ha desarrollado en otras obras el tema de la acción humana, utilizando un vocabulario distinto de británicos como lo son Archer y Hampshire y destacando la imaginación como facultad típicamente humana. 
cular. En fin, no sabemos cuántas causalidades hay. En parte por eso el pensamiento tiene que ser complejo. Entonces, si contamos con la causalidad humana como un cuarto tipo de causa, estamos reconociendo el principio de la complejidad.

\section{Conclusión}

He intentado sugerir que la economía de comunión y los demás movimientos para reconstruir la economía con criterios éticos, se pueden enfocar como proyectos de integración social. He planteado también que aquella ciencia que pretende aportar a la integración debe contar entre sus categorías teóricas y metodológicas con elementos humanistas. Debe considerar elementos teleológicos como las finalidades y las funciones, debe considerar dentro del marco del pensamiento complejo la causalidad humana, tanto las acciones de las personas como las consecuencias producidas por las normas que constituyen y rigen las instituciones.

\section{Bibliografía}

Archer, Margaret (2000): Being Human: the problem of agency. Cambridge, Cambridge Univesity Press, 2000.

Anscombe, "Modern Moral Philosophy", Philosophy. Vol. 33 (1958) pp. 1-19; Tomás Abraham, Batallas Éticas. Buenos Aires: Nueva Visión, 1995.

Beckner, M. (1969): The Biological Way of Thought. New York, Columbia University Press.

Beckner, M. (1969): "Function and Teleology", Journal of the History of Biology. Vol. 2 (pp. 151-164).

Booth, Alan; Edwards, John; Johnson, David (1991): “Social Integration and Divorce", en Social Forces, Tomo 70, No. 1 (Sep. 1991) (pp. 207-224).

Breault, K.D. (1986): "Suicide in America, an Empirical Test of Durkheim's Theory of Religious and Family Integration", American Journal of Sociology, Tomo 93, No. 2 (Nov. 1986) (pp. 628-656). 
Cassasus, Juan (2001): "Los sistemas de ideas, el poder y la identidad", en Martinic, Sergio y Prado, Marcela (eds.): Economía Política de las Reformas Educativas en América Latina. Santiago, CIDE/OREAL (pp. 2-341).

Coraggio, José Luis (2004): De la Emergencia a la Estrategia. Buenos Aires, Espacio.

Durkheim, Emile (1930) [1893]: De la division du travail social. París: Presses Universitaires de France.

Durkheim, Emile (1902) [1893]: De la division du travail social. París: Felix Alcan.

Durkhiem, Emile (1930) [1897]: Le suicide. París: Libraire Felix Alcan.

Edwards, Verónica (1990): Los Sujetos y la Construcción Social del Conocimiento Escolar en Primaria: un estudio etnográfico. Santiago, PIIE.

Etzioni, Amitai (1994): The Spirit of Community: The Reinvention of American Society. New York: Simon and Schuster.

Giddens, Anthony (1971): Capitalism and Modern Social Theory: An Analysis of the Writings of Marx, Durkheim, and Max Weber: Cambridge UK: Cambridge University Press.

Glassner, Barry y Berg, Bruce (1980): "How Jews Avoid Alcohol Problems", en American Sociological Review. Tomo 45, No. 4 (Agosto 1980) (pp. 647-664).

Gold, Lorna (2004): "The Economy of Communion: a case study of business and civil society partnership for change". Development in Practice. Vol. 14.

Gold, Lorna (2003): “The Roots of the Focolare Movement's Economic Ethics", Journal of Markets and Morality. Vol. 6 (pp. 14359).

Habermas, Jurgen (1975): Conocimiento e Interés. Buenos Aires, Amorrortu.

Hartjen, Clayton A. (1982): “Delinquency, Development, and Social Integration in India". En Social Problems. Tomo 29, No. 5 (Jun. 1982), (pp. 464-473).

Hart, H.L.A. (1962): The Concept of Law. Oxford, Clarendon Press.

Landecker, Werner (1951): "Types of Integration and their Measurement", American Journal of Sociology, Tomo 56, No. 4 (enero 1951), (pp. 332-340). 
Lubich, Chiara (1948): "Erano un cuor solo e una anima sola", Amico Serafico (pp. 236-37).

Lubich, Chiara (2003): La Economía de Comunión, Historia y Profecía. Buenos Aires, Ciudad Nueva.

Meillassoux, Claude (1981): Maidens, Meal, and Money. Cambridge, Cambridge University Press.

Morin, Edgar (1990): Introductión a la pensée complexe. París: ESF.

Nelson, G.; Lord, J. y Ochocka, J. (2001): Shifting the Paradigm in Community Mental Health: towards empowerment and community. Toronto: University of Toronto Press.

Pérez Adán, J. (1997): Socioeconomía. Madrid, Trotta.

Piaget, Jean (1932): Le jugement morale chez l'enfant. París: Presses Universitaires de France.

Polanyi, Karl (2003) [1944]: La Gran Transformación. Los orígenes políticos y económicos de nuestro tiempo, México D.F., Fondo de Cultura Económica, México.

Prahalad, C.K (2005): La Fortuna en la Base de la Pirámide. Madrid, Granica.

Radcliffe-Brown, A.R. (1965): Structure and Function in Primitive Societies. New York: Free Press.

Razeto, Luis (2000): Las Empresas Alternativas. Montevideo, Nordam.

Richards, Howard (2008): Solidaridad, Participación, Transparencia: conversaciones sobre el socialismo en Rosario, Rosario, Fundación Estévez Boero.

Richards, Howard (1964): "Deference", International Journal of Ethics. Volumen 74 (pp. 135-142).

Smith, Adam (1976) [1776]: The Wealth of Nations. Oxford: Clarendon Press.

Spencer, Herbert (1851): Social Statics. London: John Chapman, 1851.

Swedberg, Richard (1998): Max Weber and the Idea of Economic Sociology. Princeton: Princeton University Press.

Tironi, Eugenio (2005): El Sueño Chileno. Comunidad, familia y nación en el bicentenario. Santiago, Taurus.

Turner, Jonathan (1981): "Durkheim's Theory of Integration in Differentiated Social Systems". The Pacific Sociological Review. Tomo 24, No. 4 (octubre 1981) pp. 379-391. 
Integración Social y Economía de Comunión - Howard Richards

Varela, Francisco, y Maturana, Humberto (1972): “Mechanism and Biological Explanation", Philosophy of Science. Vol. 39 (pp. 378-382). 